\title{
Profound vision loss impairs psychological well-being in young and middle-aged individuals
}

\author{
This article was published in the following Dove Press journal: \\ Clinical Ophthalmology \\ 22 February 2017 \\ Number of times this article has been viewed
}

\author{
Giancarlo A Garcia ${ }^{1,2}$ \\ Matin Khoshnevis ${ }^{1,3}$ \\ Jesse Gale ${ }^{1,4}$ \\ Starleen E Frousiakis ${ }^{1,5}$ \\ Tiffany J Hwang ${ }^{1,6}$ \\ Lissa Poincenot ${ }^{1}$ \\ Rustum Karanjia 1,7-9 \\ David Baron ${ }^{6}$ \\ Alfredo A Sadun ${ }^{1,7}$ \\ 'Doheny Eye Institute, Los Angeles, \\ CA, USA; ${ }^{2}$ University of California, \\ Irvine School of Medicine, Irvine, CA, \\ USA; ${ }^{3}$ Department of Ophthalmology, \\ Temple University, Philadelphia, PA, \\ USA; ${ }^{4}$ Wellington School of Medicine \\ and Health Sciences, University of \\ Otago, Wellington, NZ; ${ }^{5}$ Department \\ of Ophthalmology, New York \\ Medical College, Valhalla, NY, USA; \\ ${ }^{6}$ Department of Psychiatry \& The \\ Behavioral Sciences, Keck School \\ of Medicine, University of Southern \\ California, Los Angeles, CA, USA; \\ ${ }^{7}$ Doheny Eye Centers, Department of \\ Ophthalmology, David Geffen School \\ of Medicine at UCLA, Los Angeles \\ California, CA, USA; ${ }^{8}$ Department \\ of Ophthalmology, University of \\ Ottawa, Ottawa, ON, Canada; ${ }^{9}$ Ottawa \\ Hospital Health Research Institute, \\ Ottawa, ON, Canada
}

Purpose: The aim of this study was to evaluate the effects of profound vision loss on psychological well-being in adolescents, young adults, and middle-aged adults with regard to mood, interpersonal interactions, and career-related goals. In addition, we assessed the significance of the resources that may be used to enhance psychological well-being in cases of profound vision loss, and in particular, examined the utility of low vision aids and the role of the ophthalmologist as a provider of emotional support.

Methods: A questionnaire was issued to individuals aged 13-65 years with profound vision loss resulting from Leber's hereditary optic neuropathy (LHON). Depression prevalence was evaluated with questions regarding major depressive disorder symptomatology. Participants appraised the effects of vision loss on their interpersonal interactions and career goals by providing an impact rating (IR) on a 21-point psychometric scale from -10 to +10 . Social well-being index was defined as the average of interpersonal IR and career IR. Subjects were additionally asked about the use of low vision aids and sources of emotional support.

Results: A total of 103 participants (mean age $=26.4 \pm 11.2$ years at LHON diagnosis; mean \pm standard deviation) completed the questionnaire. Nearly half (49.5\%) met the depression criteria after vision loss. Negative impacts on interpersonal interactions (median IR $=-5$ ) and career goals (median IR $=-6$ ) were observed; both ratings were worse $(P<0.001)$ for depressed versus nondepressed subjects. Older age at diagnosis corresponded to higher depression prevalence and increased incidence of negative interpersonal IR and career IR. Sixty-eight percent of subjects used electronic vision aids; controlling for age, social well-being index was higher among these individuals than for those who did not use electronic aids $(P=0.03)$. Over half of the participants $(52.4 \%)$ asserted that they derived emotional support from their ophthalmologist.

Conclusion: Profound vision loss in adolescents, young adults, and middle-aged adults is associated with significant negative psychological and psychosocial effects, which are influenced by age and use of electronic vision aids. Ophthalmologists, in addition to managing vision loss, may serve an important role in the emotional adaptation of these patients.

Keywords: vision loss, psychological, depression, low vision aids, quality of life, Leber's hereditary optic neuropathy

\section{Introduction}

Vision loss can be a traumatic life event. Care of patients experiencing vision loss requires attention to needs that extend beyond the immediate ocular concerns. In the elderly (aged 65 years and older), the negative psychological consequences of vision loss resulting from glaucoma, age-related macular degeneration, cataracts, and diabetic retinopathy have been well established. ${ }^{1-7}$ However, there is a dearth of literature on the psychological effects of visual disability in younger individuals. In adults aged
Correspondence: Giancarlo A Garcia Doheny Eye Institute, 800 South Fairmount Avenue, Suite 215, Pasadena, CA 91105, USA

$\mathrm{Tel}+\mathrm{I} 626817470 \mathrm{I}$

Fax +l 6268174702

Email giancarg@uci.edu
Clinical Ophthalmology 2017:II 417-427

417

Dovepress in

http://dx.doi.org/| 0.2147/OPTH.SII3414 (c) (1) (\$) 2017 Garcia et al. This work is published and licensed by Dove Medical Press Limited. The full terms of this license are available at https://www.dovepress.com/terms.php cc. hereby accept the Terms. Non-commercial uses of the work are permitted without any further permission from Dove Medical Press Limited, provided the work is properly attributed. For permision for commercial use of this work, please see paragraphs 4.2 and 5 of our Terms (https://www.dovepress.com/terms.php). 
20 years and older, vision loss has been associated with increased risk of depression, ${ }^{8,9}$ though the differences in psychological responses among different age-groups within this age demographic - and, importantly, the psychological consequences among adolescents - have not been well characterized. Furthermore, the impact on other important components of well-being - particularly interpersonal relationships and career - has not been thoroughly evaluated. An understanding of the psychological and psychosocial effects of visual disability among younger adults and adolescents is of particular importance, as members of these age-groups face unique emotional concerns, social challenges, and careerrelated pursuits that may not be present among the elderly.

Leber's hereditary optic neuropathy (LHON) may present a unique model for the examination of the psychological consequences of visual impairment in younger individuals. LHON is an inherited mitochondrial disorder that can lead to profound sequential central vision loss - typically over a period of 6-8 weeks - usually resulting in legal blindness. ${ }^{10}$ After experiencing vision loss in one eye, 97\% of affected individuals experience an inter-eye delay ranging from days to 1 year before the fellow eye is affected. ${ }^{11-13}$ Estimated mean age of onset is 24.3 years among males and 31.3 years for females; however, the age of onset can be highly variable, ranging from early childhood to ages $>50$ years. ${ }^{14}$ Various factors may hasten the onset interval - particularly smoking and use of alcohol ${ }^{15}$ - though patients' age has not been definitively correlated with the speed of onset. Young adult males are most commonly affected, with an approximate 4.5:1 ratio of affected males to females. ${ }^{16}$

An understanding of the patterns of psychological adjustment and coping in these circumstances may be relevant to the comprehensive care of patients with vision loss. LHON affects a wide age range within this younger demographic and thus provides an opportunity to elucidate the potential differences in how vision loss is dealt with among different age-groups. In addition, LHON may lend insights into the potential magnitude of the psychological impact of blindness, as the rapid onset of vision loss - combined with limited treatment options - has the potential for being particularly traumatic. The psychological and psychosocial effects of vision loss from LHON were evaluated in adolescents, young adults, and middle-aged adults with regard to mood, interpersonal interactions, and career goals. It was hypothesized that such visual disability has a negative impact on these elements and, further, that different effects may be observed in younger individuals compared with older individuals. In addition, the authors assessed the significance of the resources that may be used to enhance psychological well-being in cases of profound vision loss, and, in particular, examined the utility of low vision aids and the role of the ophthalmologist as a provider of emotional support.

\section{Methods}

\section{Study design}

The University of Southern California Institutional Review Board (Los Angeles, CA, USA) approved this study. This study was carried out in accordance with the Declaration of Helsinki and complied with the Health Insurance Portability and Accountability Act. Subjects were recruited via electronic invitations to an anonymous online questionnaire. Individuals affected by LHON were recruited from several sources to survey a broad spectrum of individuals with the disease. These sources included: 1) patients at a neuro-ophthalmology practice in southern California (Doheny Eye Institute, Los Angeles, CA, USA), 2) an email list provided by the United Mitochondrial Disease Foundation (UMDF) of patients with LHON who had signed up for the UMDF.org newsletter, and 3) an online social network for individuals with LHON. Of note, the LHON social networking forum was not specifically designed for or advertised as a forum for psychological or emotional support, but rather as a platform for patients to interact and connect with other individuals with similar visual disability.

The survey was issued to 267 individuals affected by LHON using Health Insurance Portability and Accountability Act-compliant software (Qualtrics, Provo, UT, USA) between December 2014 and May 2015. These individuals were provided with a link to complete the online questionnaire and informed that their participation and responses were made anonymous. To ensure participant anonymity, the link to the survey was distributed by a research technician; the authors of the study were made aware of the total number of individuals recruited but were blinded to the proportions of individuals recruited from each of the three recruitment sources. Due to the anonymous nature of this survey, requirement for informed consent was waived by the the University of Southern California Institutional Review Board (Los Angeles, California, USA). All survey instructions and questions were written in English. Subjects were asked to provide their age, sex, ethnicity, approximate dates of onset of vision loss and diagnosis of LHON, laterality of vision loss, best-corrected visual acuity (BCVA) in the better-seeing eye before the onset of rapid profound vision loss (which is referred to as "premorbid BCVA"), and BCVA after the onset of rapid profound vision loss. Subjects were included in the study if they were 1) between the ages of 13 years and 65 years, inclusive; 2) fluent in English; and 3) had vision loss resulting from LHON diagnosed by an ophthalmologist. Subjects were excluded if they did not 
complete the questionnaire in its entirety. For the purposes of this study, profound vision loss was defined as BCVA of 20/500 or worse, in accordance with the criteria of the American Medical Association. ${ }^{17}$

\section{Model of psychological well-being}

The model of psychological well-being employed in this investigation is outlined in Figure 1. Profound vision loss serves as the primary physiological stressor in this model. Psychological well-being has three components in this model: mood (affect), quality of an individual's interpersonal interactions, and career goals. Interpersonal interactions comprise social participation and relationships both inside and outside of one's household. Career goals represent aspirations and motivation for subjective career success within one's current career or desired future vocation. This is a future-oriented paradigm, rather than an assessment of happiness with current career status, as the latter can vary between individuals regardless of outside influences. Furthermore, this forwardthinking assessment of career goals accounts for individuals who are not employed or do not yet have an established career, which may be particularly relevant among younger individuals. These components of psychological well-being may also influence one another and likely contribute to the overall quality of life. Although other factors can impact psychological well-being, the three elements employed in this model were of particular interest in the analyses because of their central importance in long-term psychological health, and because of their susceptibility to profound change with vision loss.

\section{Assessment of psychological well-being}

Premorbid psychological health was evaluated by asking subjects whether they had experienced any mental health concerns or had visited a mental health professional prior to the onset of

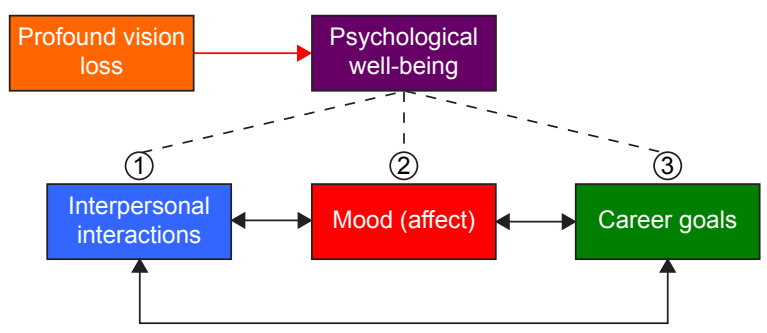

Figure I Illustrative model depicting the components of and factors influencing psychological well-being.

Notes: In this model, psychological well-being is divided into three components, as indicated by dashed lines: I, interpersonal interactions; 2, mood (affect); and 3 , career goals. The primary physiological stressor in this model, profound vision loss, may influence psychological well-being (unidirectional red arrow). Bidirectional black arrows indicate potential effects of each component on one another. For example, mood or affect may impact interpersonal life, and such an effect may influence mood or affect. vision loss. Subjects who responded affirmatively were asked to specify the nature of the mental health concerns and/or the type of mental health specialist. To assess the prevalence of depressive symptoms after the onset of vision loss, subjects were posed a series of questions in accordance with the criteria for major depressive disorder (MDD) from the Diagnostic and Statistical Manual of Mental Disorders- $V .{ }^{18}$ Participants were asked to state whether they had experienced any of the following during the 2-week period immediately prior to taking the survey: 1) depressed mood, 2) anhedonia, 3) feelings of guilt or worthlessness, 4) fatigue, 5) changes in appetite, 6) sleep disturbances, 7) psychomotor retardation or agitation, 8) difficulty concentrating, and 9) suicidal ideations. These were explained in lay terminology, for example, anhedonia was described as "loss of interest or pleasure in doing things you previously enjoyed". Participants who acknowledged experiencing at least five of these nine symptoms - with one being either depressed mood or anhedonia - were deemed to have presumed depression, which is consistent with but not definitive for MDD.

Participants were also asked to appraise the effects of vision loss on the quality of their interpersonal interactions and career goals. For each of these two categories, subjects were instructed to select an impact rating (IR) on a 21-point Likert-type psychometric response scale. The utility of such a 21-point scale for both respondents and researchers has previously been demonstrated. ${ }^{19,20}$ This ordinal bidirectional scale has anchor points of -10 (profound negative impact) and +10 (profound positive impact), with the midpoint value of zero corresponding to "neutral impact". These two ratings were termed interpersonal IR and career IR. For each participant, an average of these two ratings was calculated. This value, which was termed social well-being index, is a numerical representation of the effects on the social components of an individual's psychological well-being.

\section{Resources enhancing psychological well-being}

Participants were asked which types of clinicians they had seen after vision loss and which of these clinicians best provided them emotional support after vision loss. Subjects were also asked to list the nonclinicians who best provided them emotional support after vision loss. This portion of the survey specifically inquired about emotional support due to the direct relevance of emotions to the mood component of psychological well-being; mood is fundamentally a manifestation of how emotions are regulated. ${ }^{21}$ A specific definition of emotional support was not provided to subjects. Participants were additionally asked to list one or more low 
vision aids or assistive devices they felt were most effective in enhancing their quality of life.

For these questions, multiple-choice response categories were provided to participants to maintain independence of categories (eg, to avoid having a subject write an open-ended response of "family member" when he or she in fact meant "spouse"). Categories were listed in alphabetical order, and participants were instructed to choose one or multiple responses. However, to minimize response bias, for each of these questions, an open-ended response category of "other (specify)" was included as well.

\section{Statistical analysis}

Statistical analyses were performed using Statistical Package for Social Sciences 22.0 (IBM Corporation, Armonk, NY, USA). Mean values were calculated with corresponding standard deviation. All Snellen BCVA data were converted to logarithm of the minimum angle of resolution (logMAR) units, with the values 2.6 and 2.7 assigned to the visual acuities "count fingers" and "hand motion", respectively. ${ }^{22}$ Agegroups were defined as follows: adolescents (13-20 years), young adults (21-40 years), and middle-aged adults (41-65 years). Young adults were further divided into two groups (21-30 years and 31-40 years) for additional agerelated statistical comparisons.

Differences in proportions for two categorical variables were evaluated using Pearson's chi-squared test or with Fisher's exact test when chi-square was not appropriate. Ordinal data (IRs and social well-being index) were analyzed using the Mann-Whitney $U$ test (Wilcoxon rank sum test). Variances were compared using the nonparametric Levene's test for homogeneity of variances. Effect sizes for the difference between two sample medians were determined by dividing the Mann-Whitney $U$ test statistic by the product of the two sample sizes. An age-matched subgroup of 27 subjects from the sample was selected to control for age differences among subjects who used electronic aids and those who did not. Independent sample $t$-tests were used to evaluate differences in age among sample subgroups. An alpha level of 0.05 was used to determine statistical significance for the analyses.

\section{Results}

\section{Study population}

A total of 267 individuals with vision loss resulting from LHON were issued the survey, and 116 of these individuals participated (response rate $43.4 \%$ ). Of the 116 participants (11.2\%), 13 did not complete all components of the questionnaire, and thus were excluded from further analyses. Due to the anonymous nature of the survey, the number of individuals from each recruitment source who completed the survey could not be determined. Table 1 shows the demographic and clinical characteristics of the 103 participants who completed the survey in its entirety. All subjects reported no significant visual impairment before the onset of LHON. Mean premorbid logMAR BCVA was $0.1 \pm 0.03$ (mean \pm standard deviation), corresponding to a Snellen equivalent of $\sim 20 / 24$. All participants reported bilateral visual impairment at the time of the survey, with a BCVA range of 20/60 - hand motion, and a mean logMAR BCVA of 1.6 \pm 0.4 , corresponding to a Snellen equivalent of $\sim 20 / 796$.

Mean BCVA at the time of the survey was significantly worse compared with premorbid BCVA $(P<0.001)$.

The mean age of participants was $26.4 \pm 11.2$ years (range 14-65 years) at the time of diagnosis of LHON. In the agematched control subgroup, the age at the time of survey was not statistically significantly different $(P=0.36)$ between the electronics users (mean $=33.1 \pm 6.1$ years, $n=15$ ) and those who used exclusively nonelectronic aids or no aids ( mean $=37.0 \pm 4.0$ years, $n=12$ ).

The majority of participants $(92.2 \%, 95 / 103)$ denied a history of premorbid mental health concerns, with $7.8 \%(8 / 103)$

Table I Participant demographic and clinical characteristics ( $N=103)$

\begin{tabular}{ll}
\hline Characteristic & $\begin{array}{l}\text { n (\%) or mean } \pm \\
\text { standard deviation }\end{array}$ \\
\hline Sex & $80(77.7)$ \\
Male & $23(22.3)$ \\
Female & $24.5 \pm 10.2$ \\
Age at the onset of vision loss, years & $26.4 \pm 11.2$ \\
Age at the diagnosis of LHON, years & $29.5 \pm 13.2$ \\
Age at the time of survey, years & $5.0 \pm 1.3$ \\
Time from vision loss onset to survey, years & \\
Age range at the diagnosis of LHON, years & $38(36.9)$ \\
I3-20 & $4 I(39.8)$ \\
2 I-30 & $9(8.7)$ \\
3 I-40 & $15(14.6)$ \\
4 I-65 & \\
Race/ethnicity & $86(83.5)$ \\
Non-Hispanic White & $7(6.8)$ \\
Hispanic & $6(5.8)$ \\
Asian & $2(1.9)$ \\
Pacific Islander & $2(1.9)$ \\
Black/African American & $0.1 \pm 0.03$ \\
Premorbid BCVA, logMAR & $1.6 \pm 0.4$ \\
BCVA at the time of survey, ${ }^{*}$ logMAR &
\end{tabular}

Notes: Premorbid BCVA refers to visual acuity before the onset of rapid profound vision loss. $* P<0.001$ compared with premorbid $B C V A$.

Abbreviations: BCVA, best-corrected visual acuity; LHON, Leber's hereditary optic neuropathy; logMAR, logarithm of the minimum angle of resolution; SEM, standard error of the mean. 
reporting having mental health-related complaints before the onset of vision loss. These included depression $(3.9 \%$, $4 / 103)$, anxiety $(1.9 \%, 2 / 103)$, seasonal changes in affect $(1.0 \%, 1 / 103)$, and prior concussions $(1.0 \%, 1 / 103)$. These were the only individuals $(7.8 \%, 8 / 103)$ who had seen a mental health professional before losing vision; $4.9 \%$ $(5 / 103)$ had seen a psychologist and 3.9\% (4/103) had seen a psychiatrist.

\section{Impact on psychological well-being}

Nearly half of the participants $(49.5 \%, 51 / 103)$ met the criteria for presumed depression after vision loss, while $50.5 \%$ $(52 / 103)$ did not. There was no statistically significant difference in the percentage of males $(47.5 \%, 38 / 80)$ versus females $(56.5 \%, 13 / 23)$ fulfilling depression criteria $(P=0.45)$.
The distributions of individuals' subjective assessment of the impact of vision loss on their interpersonal life and career goals are shown in Figure 2. Median interpersonal IR was -5. A total of $70.9 \%$ of all participants (73/103) selected a negative interpersonal IR, $16.5 \%$ of all participants (17/103) selected a positive interpersonal IR, and $12.6 \%$ of all participants $(13 / 103)$ indicated a neutral impact on their interpersonal interactions. Of the participants who selected a non-neutral value, $81.1 \%$ (73/90) described a negative impact, and 18.9\% (17/90) designated a positive impact (Figure 2A).

With regard to impact on career goals, median career IR was -6 . A total of $73.8 \%$ of all participants (76/103) selected a negative interpersonal IR, $22.3 \%$ of all participants (23/103) selected a positive interpersonal IR, and 3.9\% of all participants (4/103) indicated a neutral impact on career
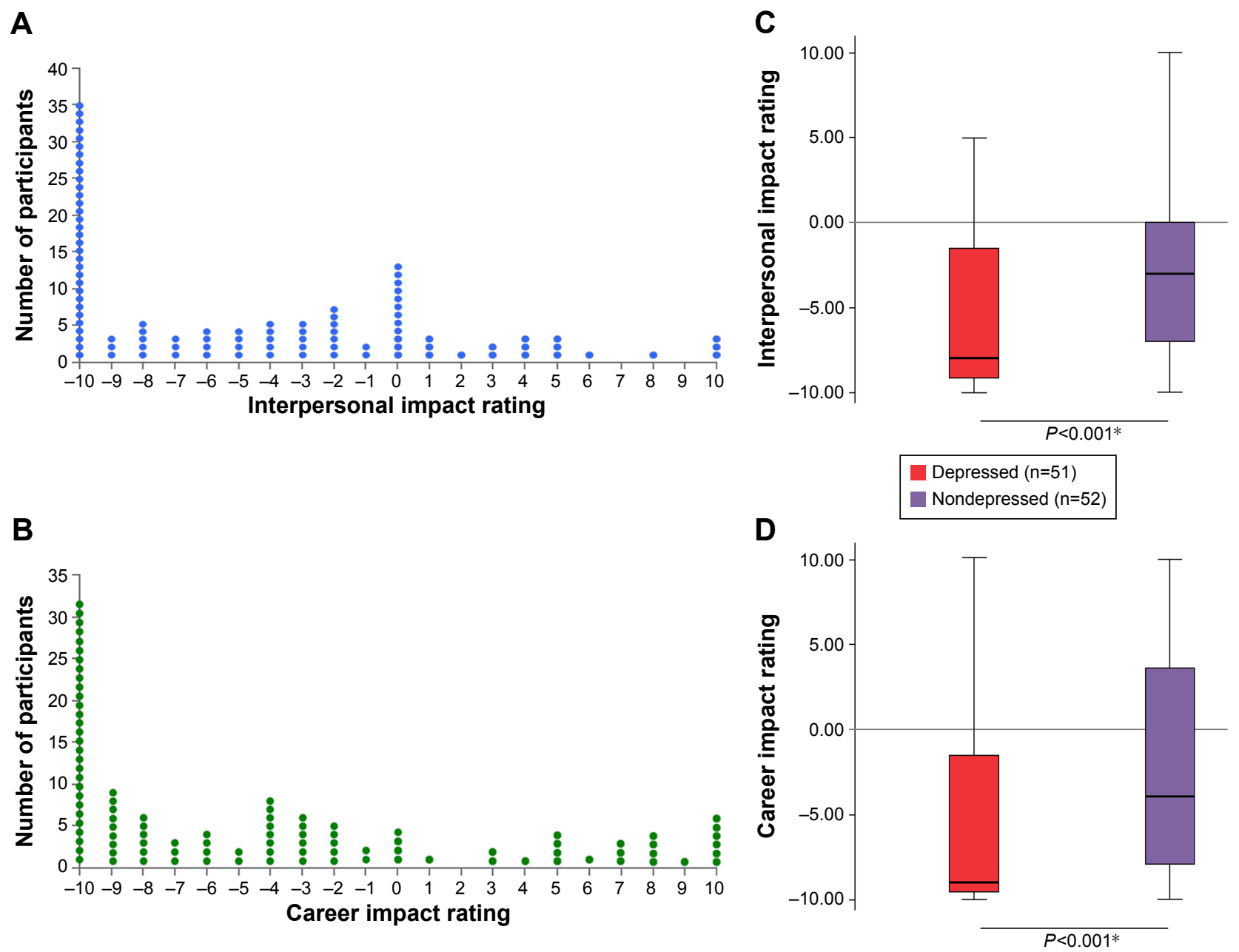

Figure 2 Interpersonal and career impact ratings.

Notes: Subjective IR distributions for interpersonal interactions (A) and career goals (B) among 103 participants. Each circle in these distributions corresponds to the impact rating of one participant. Among subjects with presumed depression, median interpersonal IR (C) and career IR (D) were statistically significantly lower $(* P=0.00 \mathrm{I}$ and $* P<0.00$ I, respectively) than among those who did not meet criteria for presumed depression. The boxes define the IQR between the $Q$ I and $Q 3$. Median values are represented by a horizontal black line inside each box. Each vertical line extends from the minimum to the maximum value. Outliers were defined as values that exceed $\mathrm{Q} 3$ by $>$ I.5.IQR or that lie >I.5.IQR beneath QI. No outliers were observed in these data sets. The $P$-values are determined by the Mann-Whitney U test. Abbreviations: IQR, interquartile range; IR, impact rating; QI, first quartile; Q3, third quartile. 
goals. Of the respondents selecting a nonneutral value, $76.8 \%$ (76/99) expressed a negative impact, and 23.2\% (23/99) noted a positive career IR (Figure 2B).

Approximately $70.9 \%$ of all participants (73/103) indicated a negative impact on both interpersonal life and career goals versus $16.5 \%(17 / 103)$ who expressed a positive impact on both; 3.8\% (4/103) expressed a neutral impact on both indices; $2.9 \%(3 / 103)$ indicated a negative career IR with a neutral interpersonal IR; and 5.8\% (6/103) reported the inverse. No individuals indicated both a positive rating on one index and negative rating on the other. The maximal negative rating ( -10 , profound negative impact) was selected by $34.0 \%$ of respondents $(35 / 103)$ in regard to interpersonal interactions and $30.1 \%$ of individuals $(31 / 103)$ with respect to career goals.

Interpersonal IR was significantly lower $(P<0.001)$ among individuals with presumed depression ( median $=-8$ ) compared with those who did not meet depression criteria (median $=-3$; Figure 2C). Similarly, career IR was significantly lower $(P<0.001)$ for participants with presumed depression $($ median $=-9$; Figure $2 \mathrm{D})$ compared with nondepressed individuals (median $=-4$ ). These differences corresponded to moderate effect sizes of 0.32 for interpersonal IR and 0.35 for career IR.

\section{Effects of age}

A significantly higher prevalence of presumed depression and higher incidences of negative interpersonal IR and negative career IR were noted among subjects who were older when diagnosed with vision loss secondary to LHON $(P<0.05$ for each measure; Figure $3 \mathrm{~A}-\mathrm{C}$ ). The highest percentages of these measures were among the age-groups of 31-40 years and 41-65 years, with no significant differences between the two $(P>0.05$ for each measure; Figure $3 \mathrm{~A}-\mathrm{C})$. For each measure, a statistically significantly lowest percentage was observed in the adolescent age-group (13-20 years).

\section{Resources enhancing psychological well-being}

After vision loss, subjects visited the following clinicians: general ophthalmologist (100\%, 103/103), neuro-ophthalmologist $(100 \%, 103 / 103)$, optometrist $(79.6 \%, 82 / 103)$, family practitioner $(75.7 \%, 78 / 103)$, genetic counselor $(22.3 \%, 23 / 103)$, psychologist $(19.4 \%, 20 / 103)$, and psychiatrist $(11.7 \%$, 12/103). Approximately $8.7 \%$ of individuals (9/103) indicated other (specify); none of these responses were similar to one another. These included: emergency doctor, low vision specialist, physical therapist, Bowen therapist, sleep specialist, occupational therapist, biotherapist, registered nurse, and ophthalmic technician.

Approximately $52.4 \%$ of subjects (54/103) indicated that they received emotional support from interactions with ophthalmologists; neuro-ophthalmologists and general ophthalmologists were the most commonly cited health care professionals who provided such support, as noted by $32.0 \%$ (33/103) and 20.4\% (21/103) of individuals, respectively. Only $19.4 \%$ of all respondents reported seeing a psychologist and $11.7 \%$ reported seeing a psychiatrist after vision loss. Of those who had seen a psychologist, 50\% (10/20) reported that a psychologist provided emotional support. Among those who had seen a psychiatrist, 41.7\% (5/12) indicated that a psychiatrist was emotionally supportive. Additional clinician sources of emotional support included genetic counselor $(39.1 \%, 9 / 23)$, family practitioner $(19.2 \%, 15 / 78)$, optometrist $(6.1 \%, 5 / 82)$, occupational therapist $(100 \%, 1 / 1)$, low vision specialist $(100 \%, 1 / 1)$, and sleep specialist (100\%, 1/1) among the patients who had seen these clinicians.

Family relatives $(39.8 \%, 41 / 103)$, nonrelative friends (26.2\%, 27/103), and online support groups $(24.3 \%, 25 / 103)$ were the most frequently mentioned sources of emotional support provided by nonclinicians. Approximately $17.5 \%$ of participants $(18 / 103)$ indicated that they derived no emotional support from individuals outside of the health care profession. For nonclinician sources of emotional support, $5.8 \%$ of individuals (6/103) indicated other (specify); none of these responses were similar to one another. These included: teacher, tutor, personal trainer, meditation group, veterans administration support group, and exercise group.

Figure 4A presents the types of low vision aids participants used to enhance their quality of life. Approximately $68.0 \%$ of individuals $(70 / 103)$ indicated the use of electronic vision aids versus $13.6 \%$ of participants (14/103) who used nonelectronic aids only and 18.4\% (19/103) who used no low vision aids. Among the most frequently cited electronic aids were the Apple iPad (Apple Inc., Cupertino, CA, USA) (26.2\%, 27/103), closed-circuit television (24.2\%, 25/103), and the Apple iPhone $(21.4 \%, 22 / 103)$. Screen magnification software and screen readers - in particular, ZoomText $(21.4 \%, 22 / 103)$ and Job Access With Speech (13.6\%, 14/103) - were also commonly used assistive technology. A magnifying glass was the most commonly cited nonelectronic tool $(16.5 \%, 17 / 103)$. Only $2.9 \%$ of participants $(3 / 103)$ utilized Braille as a low vision resource.

Social well-being index (Figure 4B) was higher among those who reported the use of electronic vision aids (median $=-3$ ) than among those who either used exclusively 
A

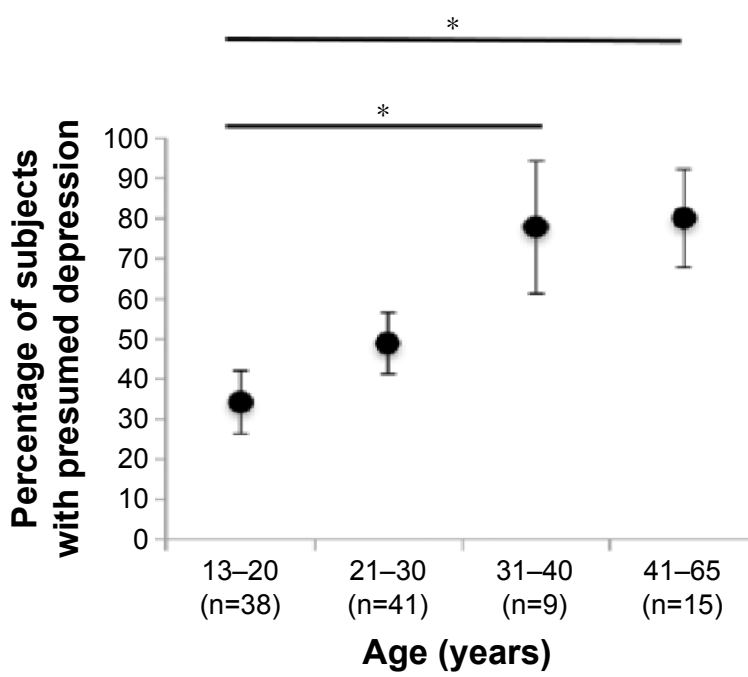

B

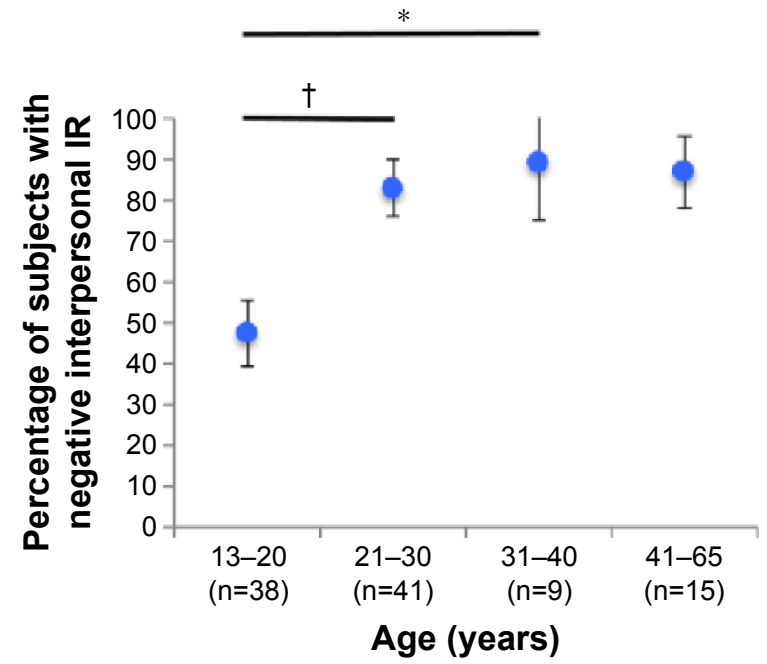

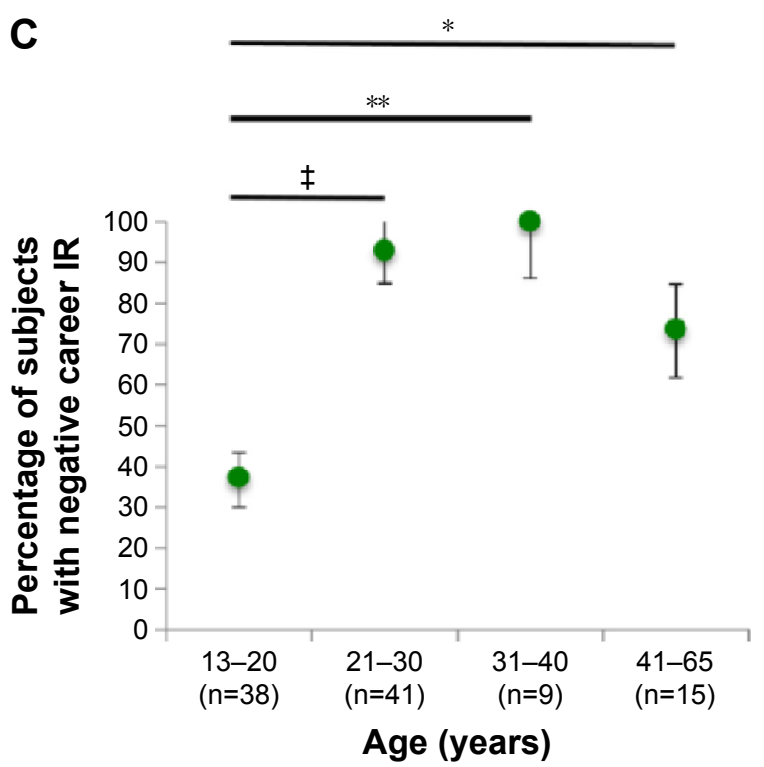

Figure 3 Percentages of subjects with presumed depression (A), negative interpersonal IR (B), and negative career IR (C) distributed by age-group at the time of diagnosis of profound vision loss secondary to LHON $(\mathrm{N}=103)$.

Notes: In all three of these distributions, percentages tend to increase as chronological age increases. Statistically significant differences among pairwise comparisons are indicated by horizontal black lines. Error bars represent standard deviation of proportion. ${ }^{\dagger} P<0.0$ I (chi-square test), ${ }^{\ddagger} P<0.00$ I (chi-square test), ${ }^{* P}<0.05$ (Fisher's exact test), $* * P<0.0$ I (Fisher's exact test).

Abbreviations: IR, impact rating; LHON, Leber's hereditary optic neuropathy.

nonelectronic aids or no low vision aids (median $=-5.5$ ). This was a statistically significant difference $(P=0.007)$ with a moderate corresponding effect size of 0.37 . The mean age at the time of survey was lower among those who utilized electronic aids (24.0 \pm 7.5 years) versus those who did not (41.0 \pm 14.6 years; $P<0.001)$. Controlling for age (age-matched control subgroup), social well-being index was still higher among those who utilized electronic low vision technology (median $=-3.5$ ) versus those who did not ( median $=-5 ; P=0.03$, effect size $=0.26$ ).

\section{Discussion}

This study demonstrates that profound vision loss can have many consequences. A thorough understanding of the psychological consequences of vision loss - and the resources that may help mitigate the potentially devastating effects - is important for the overall care of patients.

The prevalence of depression among visually impaired individuals is striking. Prior studies have demonstrated that approximately one-third or more of elderly individuals with profound vision loss may experience clinically significant 

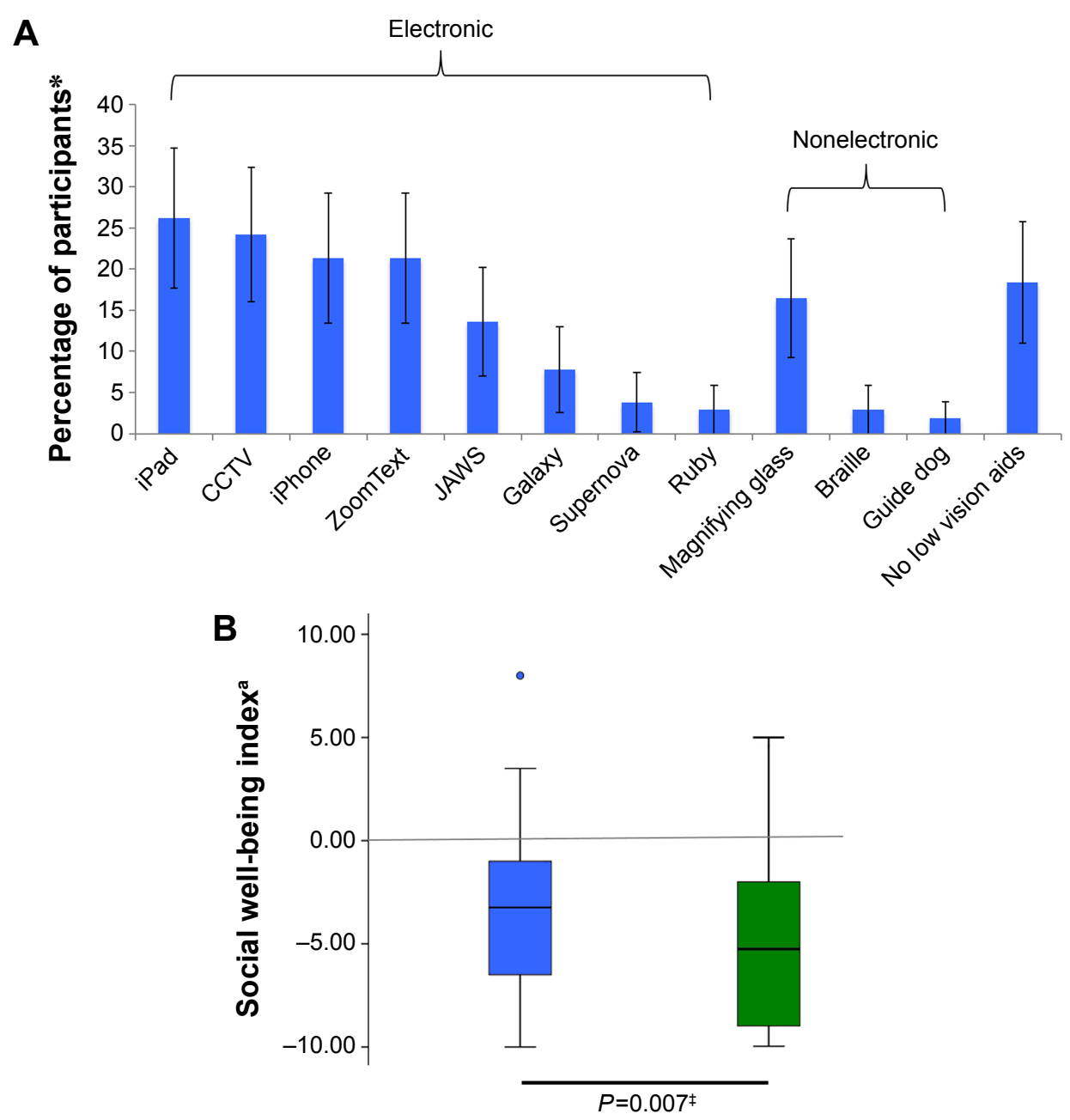

$\square$ Electronic aids ( $\mathrm{n}=71) \square$ Nonelectronic or no aids $(\mathrm{n}=32)$

Figure 4 Low vision aids used among participants and impact of electronic low vision aids on social well-being index $(\mathrm{N}=103)$.

Notes: (A) Black brackets group electronic aids (left) and nonelectronic aids (right). For purposes of clarity, only responses listed by $>$ I participant are presented. No responses of "other (specify)" were similar to one another. Approximately $6.8 \%$ of individuals (7/I03) indicated other (specify). These responses included: audiobooks, ID Mate Barcode Scanner, talking kitchen scale, Windows Magnify, binocular glasses, clip-on loupes, and white cane. Error bars are $95 \%$ confidence intervals. (B) Box-and-whisker plot diagrams depicting social well-being index. The median of this index (horizontal black line inside each box) was statistically significantly higher ( $P=0.007)$ for subjects who use electronic low vision aids (blue box, $\mathrm{n}=7 \mathrm{l}$ ) than for those who use exclusively nonelectronic aids or no vision aids (green box, $\mathrm{n}=32$ ). The data set for electronics users contains a single outlier (blue circle). iPad (tablet; Apple); CCTV (for text/image magnification); iPhone (smartphone; Apple); ZoomText (screen magnification software/screen reader; Ai Squared); JAWS (screen magnification software/screen reader; Freedom Scientific,); Galaxy (smartphone; Samsung); Supernova (screen magnification software/ screen reader; Dolphin); and Ruby (Freedom Scientific, handheld video magnifier). aSocial well-being index is calculated as the average of an individual's interpersonal impact

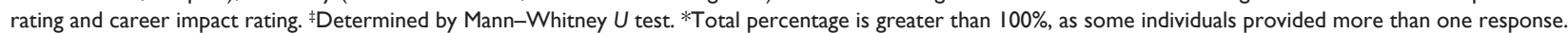
Abbreviations: CCTV, closed-circuit television; JAWS, Job Access With Speech.

depression. ${ }^{1,2,5}$ Nollett et al ${ }^{5}$ estimated that $43 \%$ of patients at a low vision rehabilitation clinic had clinically significant depression, which is nearly as high as depression prevalence in patients with cancer about to undergo chemotherapy. The present analyses revealed a similarly high prevalence of presumed depression among younger individuals, with $49.5 \%$ of participants fulfilling criteria for MDD after vision loss. The negative effects of visual disability on psychological well-being observed in this study were generally worse with increasing age. Further, the use of electronic low vision aids was associated with positive effects on psychological well-being.

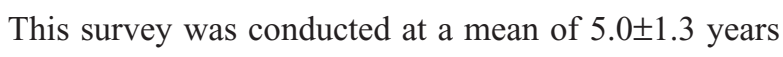
after the onset of participants' vision loss. The prevalence of MDD and impact on career and interpersonal relationships may have been different immediately following subjects' vision loss. Nonetheless, the striking proportions of individuals attesting to negative psychological and psychosocial effects highlight that ophthalmologists must recognize that these consequences can be present even several years after the onset of vision loss.

The impact of visual impairment on career and occupational goals should be of high relevance to ophthalmologists, 
although few studies have examined these effects. Rahi et $\mathrm{al}^{23}$ demonstrated that impaired vision-related quality of life was associated with inability to work and low socioeconomic status. The potentially negative impact of vision loss on career goals (Figure 2), motivation, and productivity may, in turn, have deleterious effects on income and consequent ability to afford a high level of medical care. Although many visually impaired individuals qualify for governmental health insurance programs, they may be uninsured while waiting for approval or find that certain therapies are not covered even after approval. Socioeconomic status is therefore relevant in such situations.

Clinicians caring for patients who have experienced profound visual impairment should consider a patient's age at the time of vision loss, as it may be an indicator of the potential psychological sequelae (Figure 3). In this study, individuals who were older when diagnosed with LHON reported lower levels of psychological well-being compared with their younger counterparts. Various explanations may account for these age-related differences. Younger chronological age in the context of profound vision loss may be associated with a higher potential for psychological adaptation and coping. In addition, higher incidence of negative interpersonal IR in older subjects may in part be a function of decreased social network size among older individuals. ${ }^{24}$ Furthermore, middle-aged adults - many of whom have already spent decades establishing a career - may experience a more devastating career impact compared with younger patients when faced with debilitating vision loss. The results indicated similar prevalence of presumed depression among males and females. This is consistent with prior research that has demonstrated no sex differences in incidence or severity of depression after vision loss. ${ }^{4,6}$

Data from the age-matched control subgroup indicate that, regardless of age, the use of electronic low vision aids is associated with enhanced psychological well-being (Figure 4). Haji et $\mathrm{a}^{25}$ reported that $94 \%$ of subjects with $20 / 100$ or worse visual acuity in the better eye were able to read standard size newspaper text on an iPad - versus only $22 \%$ without an iPad - lending credence to the utility of these devices. Nonetheless, the relative newness of these technologies, coupled with the paucity of literature highlighting their use as low vision aids, suggests that recommendation of these devices by ophthalmologists is not yet widespread. ${ }^{26}$ The limited popularity of nonelectronic aids among participants may be not only testament to the efficacy of electronic aids but also a consequence of potentially stigmatizing social effects. Individuals may not want to call attention to their disability by using tools such as a magnifying glass, guide dog, white cane, or Braille.

The data from this survey also underscore the potential role of ophthalmologists in providing emotional support for patients. More than half of the respondents (52.4\%) asserted that ophthalmologists were a source of emotional support. However, there is a dearth of prior data highlighting the importance of ophthalmologists themselves as potential emotional counselors for patients with profound vision loss. Importantly, the concept of emotional support varies widely among individuals,${ }^{27}$ and therefore the mechanisms by which an ophthalmologist can offer such support likely vary between patients. Possible mechanisms of emotional care offered from ophthalmologists to patients in this survey may be either direct - through active emotional counseling or attentive listening - or indirect - by offering patients definitive answers and knowledge of their condition that may have previously been unsatisfied. The latter likely explains why LHON patients more commonly cited neuroophthalmologists as the most helpful providers of emotional support, followed by general ophthalmologists.

The ability of ophthalmologists to provide emotional support could have profound implications for patient outcomes; visually impaired patients with depressed mood experience decrements in visual function that cannot be entirely accounted for by their eye disease $\mathrm{e}^{5}$ and suffer higher levels of overall disability compared with their nondepressed counterparts. ${ }^{28}$ Of note, $47.6 \%$ of participants did not cite ophthalmologists as sources of emotional support. This highlights that there are important sources of support from other types of clinicians, such as family practitioners. Nonetheless, the data underscore the significant potential for ophthalmologists in the emotional care of patients with profound vision loss, which may currently be underemphasized in eye care clinics.

Many individuals derived benefit from seeing a mental health professional. However, the role of mental health specialists in enhancing psychological well-being in patients with profound visual disability is inconclusive, as fewer than one-third of subjects (31.1\%) in this study sought care from a psychologist or psychiatrist after vision loss.

Significant vision loss may be particularly detrimental to social interactions, as individuals with poor vision may have difficulty maintaining eye contact and recognizing facial expressions, both of which are critical components of social communication. ${ }^{29,30}$ Social isolation is a risk factor for MDD, and MDD increases the risk of further social withdrawal (Figure 1). ${ }^{31}$ The IRs provided by participants 
may demonstrate the interconnectedness of these elements of psychological well-being, for example, individuals with presumed depression had more negative self-appraisals of interpersonal interactions and career goals compared with nondepressed subjects (Figure 2).

\section{Limitations}

This study has a number of limitations. First, the components of psychological well-being assessed in this analysis are not independent variables, as each component may affect one another. Other influences on psychological well-being, such as the ability to perform activities of daily living, educational pursuits, spirituality, security, and fertility, were not specifically addressed in this investigation. In addition, patient information such as age at onset of symptoms and age at diagnosis of LHON were self-reported and not verified through clinical records.

The survey in this study was designed only for English speakers and only was available to individuals with computer and Internet access; as such, this investigation may have been prone to selection and data acquisition biases. This may have influenced the analyses regarding the use of low vision aids, as individuals with access to the computer and Internet technology required to compete the survey may have been more likely to use electronic low vision aids as well. Internet access and use of electronic aids may be related to socioeconomic status and living environment (eg, urban or rural), though these factors were not specifically addressed in this study. Recruitment of subjects from the online social networking forum and the UMDF.org email list may have introduced additional selection bias, as these participants may have had stronger interest in their disease. While the social network was not specifically designed as a forum for psychological support, some individuals may have joined these networks for such purposes.

Not all subjects may have had access to mental health professionals such as psychologists and psychiatrists, and therefore conclusions about the role of these specialists in the emotional support of these patients could not be drawn. Interestingly, fewer than $1 \%$ of patients reported seeing clinicians such as low vision rehabilitation specialists or occupational therapists after vision loss. This is surprising, as patients with profound vision loss are often referred to such specialists. This unexpected finding may have been a limitation of the term "clinician" that was presented to subjects in the survey; certain participants may not have considered these specialists to be clinicians.

The results of this investigation serve as a proof of principle of the significant impairment of psychological well-being experienced by younger individuals with profound vision loss. This study utilized LHON as a model for profound vision loss, and the results may be pertinent in other causes of visual disability in younger individuals, though this remains to be definitively assessed.

The IRs provided by patients (Figure 2) may have been influenced by the magnitude of visual impairment; this was not assessed, however, as visual acuity was self-reported and not verified clinically. These ratings may also have been affected by individual differences in how patients subjectively use rating scales. The 21-point Likert-type rating scale used in this survey had only the anchor points and midpoint explicitly defined for subjects, which may have introduced extreme response or midpoint biases. Nonetheless, the overall design of this scale may mitigate this limitation. This level of scale granularity has been reported as useful to both respondents and researchers, with other iterations of a 21-point Likerttype scale demonstrating a wide range of selections among the available response options. ${ }^{19,20}$ Garner, ${ }^{32}$ for example, has suggested that maximum rating information is obtained when using scales of at least 20 response categories, and Pearse ${ }^{19}$ has pointed to the statistical rigor and accuracy that can be achieved with the 21-point scale in particular.

\section{Conclusion}

This study evaluated the consequences of profound vision loss on important aspects of psychological well-being in adolescents, young adults, and middle-aged adults, and the factors that may improve the quality of life and emotional health. While some individuals with profound vision loss may attest to a positive impact in their lives, the majority experience significant psychological morbidity. Approximately half became clinically depressed, and many reported profound negative effects on their interpersonal interactions and career goals. Ophthalmologists may play an important role in the emotional adaptation of visually impaired patients and, in particular, can emphasize the efficacy of electronic low vision aids. Further research may identify additional resources that are useful in enhancing patients' quality of life.

\section{Acknowledgments}

This research was supported by the Research to Prevent Blindness, the International Foundation for Optic Nerve Diseases, the UMDF, and LHON.org. The authors thank Jeremy Poincenot and Jonathan Dator who provided insight and expertise that significantly aided in the creation of the online questionnaire. The funding organizations had no role in the design or conduct of this research. 


\section{Disclosure}

The authors report no conflicts of interest in this work.

\section{References}

1. Cimarolli VR, Casten RJ, Rovner BW, Heyl V, Sörensen S, Horowitz A. Anxiety and depression in patients with advanced macular degeneration: current perspectives. Clin Ophthalmol. 2016;10:55-63.

2. Varano M, Eter N, Winyard S, Wittrup-Jensen KU, Navarro R, Heraghty J. The emotional and physical impact of wet age-related macular degeneration: findings from the wAMD Patient and Caregiver Survey. Clin Ophthalmol. 2016;10:257-267.

3. Yuzawa M, Fujita K, Tanaka E, Wang ECY. Assessing quality of life in the treatment of patients with age-related macular degeneration: clinical research findings and recommendations for clinical practice. Clin Ophthalmol. 2013;7:1325-1332.

4. Diniz-Filho A, Abe RY, Cho HJ, Baig S, Gracitelli CP, Medeiros FA. Fast visual field progression is associated with depressive symptoms in patients with glaucoma. Ophthalmology. 2016;123(4):754-759.

5. Nollett CL, Bray N, Bunce C, et al. High prevalence of untreated depression in patients accessing low-vision services. Ophthalmology. 2016;123(2):440-441.

6. Evans JR, Fletcher AE, Wormald RP. Depression and anxiety in visually impaired older people. Ophthalmology. 2007;114(2):283-288.

7. Brody BL, Gamst AC, Williams RA, et al. Depression, visual acuity, comorbidity, and disability associated with age-related macular degeneration. Ophthalmology. 2001;108(10):1893-1900; discussion 1900-1901.

8. Wang SY, Singh K, Lin SC. Prevalence and predictors of depression among participants with glaucoma in a nationally representative population sample. Am J Ophthalmol. 2012;154(3):436-444.e2.

9. Zhang X, Bullard KM, Cotch MF, et al. Association between depression and functional vision loss in persons 20 years of age or older in the United States, NHANES 2005-2008. JAMA Ophthalmol. 2013;131(5): 573-581.

10. Meyerson C, Van Stavern G, McClelland C. Leber hereditary optic neuropathy: current perspectives. Clin Ophthalmol. 2015;9: 1165-1176.

11. Riordan-Eva P, Sanders MD, Govan GG, Sweeney MG, Da Costa J, Harding AE. The clinical features of Leber's hereditary optic neuropathy defined by the presence of a pathogenic mitochondrial DNA mutation. Brain. 1995;118(pt 2):319-337.

12. Hotta Y, Fujiki K, Hayakawa M, et al. Clinical features of Japanese Leber's hereditary optic neuropathy with 11778 mutation of mitochondrial DNA. Jpn J Ophthalmol. 1995;39(1):96-108.

13. Newman NJ, Lott MT, Wallace DC. The clinical characteristics of pedigrees of Leber's hereditary optic neuropathy with the 11778 mutation. Am J Ophthalmol. 1991;111(6):750-762.

14. Abu-Amero KK. Leber's hereditary optic neuropathy: the mitochondrial connection revisited. Middle East Afr J Ophthalmol. 2011;18(1): $17-23$
15. Kirkman MA, Yu-Wai-Man P, Korsten A, et al. Gene-environment interactions in Leber hereditary optic neuropathy. Brain. 2009;132(pt 9): 2317-2326.

16. Sadun AA, Carelli V, Salomao SR, et al. A very large Brazilian pedigree with 11778 Leber's hereditary optic neuropathy. Trans Am Ophthalmol Soc. 2002;100:169-178; discussion 178-179.

17. Cocchiarella L, Andersson GBJ, editors. Guides to the Evaluation of Permanent Impairment. 5th ed. Chicago: American Medical Association; 2001.

18. American Psychiatric Association. Major depressive disorder. Diagnostic and Statistical Manual of Mental Disorders: DSM-5. 5th ed. Arlington: American Psychiatric Association; 2013:160-168.

19. Pearse N. Deciding on the scale granularity of response categories of Likert type scales: the case of a 21-point scale. Electron J Bus Res Meth. 2011;9(2):159-171.

20. Friedman HH, Amoo T. Rating the rating scales. J Marketing Manag. 1999;9:114-123.

21. Clark LA, Watson D, Mineka S. Temperament, personality, and the mood and anxiety disorders. J Abnorm Psychol. 1994;103(1):103-116.

22. Grover S, Fishman GA, Anderson RJ, et al. Visual acuity impairment in patients with retinitis pigmentosa at age 45 years or older. Ophthalmology. 1999;106(9):1780-1785.

23. Rahi JS, Cumberland PM, Peckham CS. Visual impairment and visionrelated quality of life in working-age adults: findings in the 1958 British birth cohort. Ophthalmology. 2009;116(2):270-274.

24. Bhattacharya K, Ghosh A, Monsivais D, Dunbar RIM, Kaski K. Sex differences in social focus across the life cycle in humans. $R$ Soc Open Sci. 2016;3:160097.

25. Haji SA, Sambhav K, Grover S, Chalam KV. Evaluation of the iPad as a low vision aid for improving reading ability. Clin Ophthalmol. 2015;9:17-20.

26. Robinson JL, Braimah Avery V, Chun R, Pusateri G, Jay WM. Usage of accessibility options for the iPhone and iPad in a visually impaired population. Semin Ophthalmol. 1-9. Epub 2015 Aug 19.

27. Antonucci TC, Johnson EH. Conceptualization and methods in social support theory and research as related to cardiovascular disease. In: Shumaker SA, Czajkowski SM, editors. Social Support and Cardiovascular Disease. New York: Springer Science+Business Media; 1994:21-36.

28. Banerjee A, Kumar S, Kulhara P, Gupta A. Prevalence of depression and its effect on disability in patients with age-related macular degeneration. Indian J Ophthalmol. 2008;56(6):469-474.

29. Kleinke CL. Gaze and eye contact. A research review. Psychol Bull. 1986;100(1):78-100.

30. Frith C. Role of facial expressions in social interactions. Philos Trans R Soc Lond B Biol Sci. 2009;364(1535):3453-3458.

31. Teo AR, Choi H, Valenstein M. Social relationships and depression: ten-year follow-up from a nationally representative study. PLoS One. 2013;8(4):e62396

32. Garner WR. Rating scales, discriminability and information transmission. Psychol Rev. 1960;67:343-352.
Clinical Ophthalmology

\section{Publish your work in this journal}

Clinical Ophthalmology is an international, peer-reviewed journal covering all subspecialties within ophthalmology. Key topics include: Optometry; Visual science; Pharmacology and drug therapy in eye diseases; Basic Sciences; Primary and Secondary eye care; Patient Safety and Quality of Care Improvements. This journal is indexed on Submit your manuscript here: http://www.dovepress.com/clinical-ophthalmology-journal

\section{Dovepress}

PubMed Central and CAS, and is the official journal of The Society of Clinical Ophthalmology (SCO). The manuscript management system is completely online and includes a very quick and fair peer-review system, which is all easy to use. Visit http://www.dovepress.com/ testimonials.php to read real quotes from published authors. 